\title{
Pulmonary arterial hypertension associated with congenital heart disease
}

\author{
Michele D'Alto* and Vaikom S. Mahadevan\#
}

\begin{abstract}
Pulmonary arterial hypertension (PAH) is a common complication of congenital heart disease (CHD), with most cases occurring in patients with congenital cardiac shunts. In patients with an uncorrected left-to-right shunt, increased pulmonary pressure leads to vascular remodelling and dysfunction, resulting in a progressive rise in pulmonary vascular resistance and increased pressures in the right heart. Eventually, reversal of the shunt may arise, with the development of Eisenmenger's syndrome, the most advanced form of PAH-CHD.

The prevalence of PAH-CHD has fallen in developed countries over recent years and the number of patients surviving into adulthood has increased markedly. Today, the majority of PAHCHD patients seen in clinical practice are adults, and many of these individuals have complex disease or received a late diagnosis of their defect. While there have been advances in the management and therapy in recent years, PAH-CHD is a heterogeneous condition and some subgroups, such as those with Down's syndrome, present particular challenges.

This article gives an overview of the demographics, pathophysiology and treatment of PAH$\mathrm{CHD}$ and focuses on individuals with Down's syndrome as an important and challenging patient group.
\end{abstract}

KEYWORDS: Congenital heart disease, Down's syndrome, Eisenmenger's syndrome, pulmonary arterial hypertension

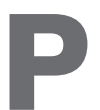
ulmonary arterial hypertension (PAH) is a frequent complication of congenital heart disease (CHD), particularly in patients with left-to-right (systemic-to-pulmonary) shunts. Persistent exposure of the pulmonary vasculature to increased blood flow and pressure may result in vascular remodelling and dysfunction. This leads to increased pulmonary vascular resistance (PVR) and, ultimately, to reversal of the shunt and development of Eisenmenger's syndrome. First described by Victor Eisenmenger in 1897 [1], Eisenmenger's syndrome was defined in 1958 as "pulmonary hypertension at systemic level due to high PVR with reversed or bidirectional blood flow through a septal defect" [2]. Eisenmenger's syndrome represents the most advanced form of $\mathrm{PAH}-$ $\mathrm{CHD}$. The current European Society of Cardiology guidelines provide an anatomical-pathophysiological classification of the congenital left-to-right shunts associated with PAH [3]. Simple defects include pre-tricuspid shunts (such as atrial septal defect (ASD)) and simple post-tricuspid shunts (including ventricular septal defects (VSD)), while complex CHD includes complete atrioventricular septal defect (AVSD), truncus arteriosus, single ventricular physiology with unobstructed pulmonary blood flow and transposition of the great arteries with VSD. In some instances, left-sided lesions can result in development of post-capillary pulmonary hypertension; however, this occurs much less frequently than $\mathrm{PAH}$ resulting from left-to-right shunts [4].

The development of PAH in patients with CHD is associated with increased mortality and high morbidity, reflected in a substantial increase in health service utilisation $[5,6]$. While successful early closure of a cardiac defect prevents the development of $\mathrm{PAH}$, and advances in paediatric cardiology and surgery have led to a marked decrease in the prevalence of PAH-CHD in western countries, the number of patients with CHD surviving into adulthood has increased $[7,8]$. However, not all patients have a fully successful repair and many continue to suffer from residual lesions and potentially serious sequelae into adulthood. Depending on their age at closure, even patients who have had a full

\section{AFFILIATIONS}

*Dept of Cardiology, Monaldi Hospital, Second University of Naples, Naples, Italy.

\#Manchester Heart Centre, Manchester Royal Infirmary, Manchester, UK.

CORRESPONDENCE

V.S. Mahadevan

Manchester Heart Centre

Manchester Royal Infirmary

Oxford Road

Manchester

M13 9WL

UK

E-mail: vaikom.mahadevan@

cmft.nhs.uk

Received:

Aug 232012

Accepted after revision:

Sept 192012

\section{PROVENANCE}

Publication of this peer-reviewed article was supported by Actelion Pharmaceuticals Ltd, Switzerland (principal sponsor, European Respiratory Review issue 126). 
repair of their cardiac defect are at risk of developing PAH. In addition, there remains a population of patients with left-toright shunts who are not diagnosed until childhood or even into adulthood. In these patients, changes to the pulmonary vasculature have already occurred, and PAH has, to a greater or lesser degree, already developed. This continually growing population of adult patients with PAH-CHD presents the clinician with a range of challenges associated with the management of complex cardiac and non-cardiac comorbidities. With the advent of PAH-specific therapies in recent years, there have been major advances in the treatment of patients with other forms of $\mathrm{PAH}$, and similar progress is beginning to be seen in PAH-CHD. However, the population of patients with PAH-CHD is heterogeneous and some subgroups present particular challenges. Among them are patients with Down's syndrome, who represent a significant and under-recognised patient group. Patients with Down's syndrome have a high incidence of complex cardiac defects and are at particular risk of developing PAH-CHD. In fact, in a recent registry study, individuals with Down's syndrome made up almost half the total population of Eisenmenger's syndrome patients [9]. Despite this, there is evidence that Down's syndrome patients are often undermanaged in terms of their PAH-CHD [9].

\section{DEMOGRAPHICS OF PAH-CHD}

The demographics of CHD are changing. Major advances in the diagnosis, management and treatment of CHD over the past few decades have led to a marked increase in the number of patients with CHD surviving into adulthood [7]. Today, the majority of patients with CHD seen in clinical practice are adults, and it is likely that they will continue to represent the largest proportion of patients requiring life-long medical care in the short-to-medium term $[10,11]$. The estimated prevalence of CHD is approximately six to 10 per 1,000 live births [12, 13] and $4-15 \%$ of patients with CHD will go on to develop PAH [14]. In the French National Registry of PAH, PAH-CHD was the second most commonly associated form of PAH (after connective tissue disease-associated PAH) [15]. Data from European registry studies give the overall prevalence of $\mathrm{PAH}$ in adult patients with CHD as $4-28 \%$ and the prevalence of Eisenmenger's syndrome as $\sim 1-6 \%[14,16]$. The prevalence of $\mathrm{PAH}$ in patients with CHD varies according to the size and location of the cardiac defect [3]. In the Dutch CONCOR registry, which included patients with both corrected and uncorrected defects, PAH prevalence rates varied from $3 \%$ in patients with patent ductus arteriosus to $100 \%$ in patients with an aortopulmonary window (fig. 1) [14]. The risk of developing Eisenmenger's syndrome also varies depending on the underlying heart defect, ranging from $\sim 10-17 \%$ in patients with an ASD (pre-tricuspid shunt), to $\sim 50 \%$ of patients with a VSD (post-tricuspid shunt), 90\% of those with unrepaired AVSD and almost all patients with truncus arteriosus [14, 17, 18]. This increase in risk clearly reflects the classification of simple (lower risk) defects with more complex (higher risk) defects [3]. However, this may not solely be a result of anatomical differences as patients with more complex defects may also have additional anomalies, such as pulmonary collaterals, which may also act as risk factors for poorer outcome (e.g. massive bleeding). It is also important not to forget that the size of the defect has an impact on the probability of developing $\mathrm{PAH}$. In this context, size not only refers to the anatomical

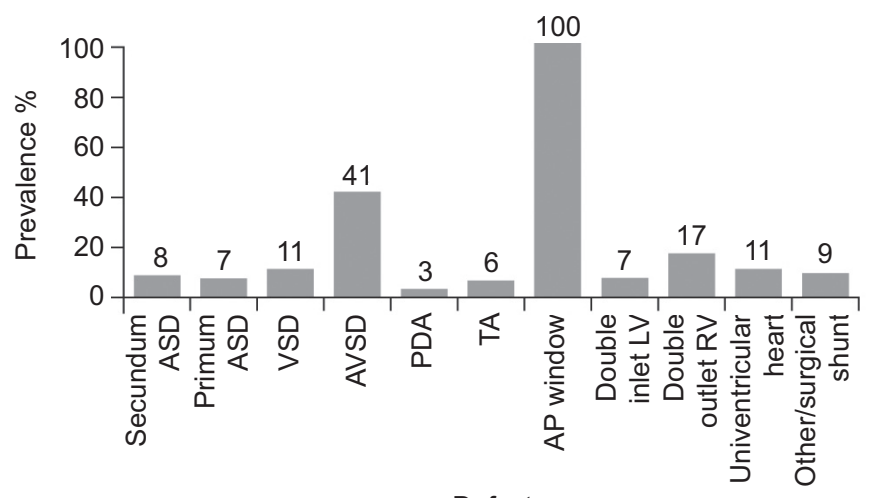

Defect

FIGURE 1. Prevalence of pulmonary arterial hypertension (PAH) in patients with congenital heart disease (CHD) according to defect. The study population includes patients with corrected and uncorrected defects. CHD: $n=2,389$. PAHCHD: $n=248$. ASD: atrial septal defect; VSD: ventricular septal defect; AVSD: atrioventricular septal defect; PDA: patent ductus arteriosus; TA: truncus arteriosus; AP: aortopulmonary; LV: left ventricle; RV: right ventricle. Data from [14].

dimensions of the defect in absolute terms, although this is important, but also to the degree of shunting, which can be assessed by measuring the pulmonary flow (Qp)/systemic flow (Qs) ratio. In general, patients with large and complex defects are at greatest risk. Patients with a post-tricuspid shunt (i.e. VSD, aortopulmonary window or complex CHD) tend to have an earlier onset of Eisenmenger's syndrome, with most patients presenting in childhood, compared with pre-tricuspid shunt patients (i.e. ASD or unobstructed anomalous pulmonary venous return) who generally do not develop pulmonary hypertension at all or present with PAH in adulthood [19]. Mortality of patients with complex anatomy (i.e. AVSD, univentricular heart, transposition of the great arteries and common arterial trunk) is higher than in those with simple anatomy (i.e. ASD, VSD or patent arterial duct). In a study of patients with Eisenmenger's syndrome at a specialist adult CHD centre, $50 \%$ of patients with simple anatomy were still alive at 58 yrs of age compared with $50 \%$ of patients with complex anatomy having died by the age of 42 yrs [20].

In the European guidelines, PAH-CHD patients have been classified into four main clinical groups (table 1) [3]. For those patients with Eisenmenger's syndrome (Group A), diagnosis is relatively straightforward and, as will be discussed, there are clinical trial data on which to base recommendations for treatment. However, for the remaining three clinical groups the situation is less defined. Group B patients with left-to-right shunts represent a more problematic population. The most common defects are ASDs [4], but other conditions, for example, partially palliated patients with unrestricted shunts or unrepaired conditions such as pulmonary atresia with major aortopulmonary collateral arteries, are increasingly being seen in the clinic. Group B patients are generally symptomatic and although many defects are not suitable for surgical repair, there is not a large evidence base to guide management decisions. Those patients with small defects who develop PAH form Group $\mathrm{C}$ of the clinical classification and have a clinical picture similar to that seen in idiopathic PAH with the advantage that the presence of a shunt (generally interatrial) 


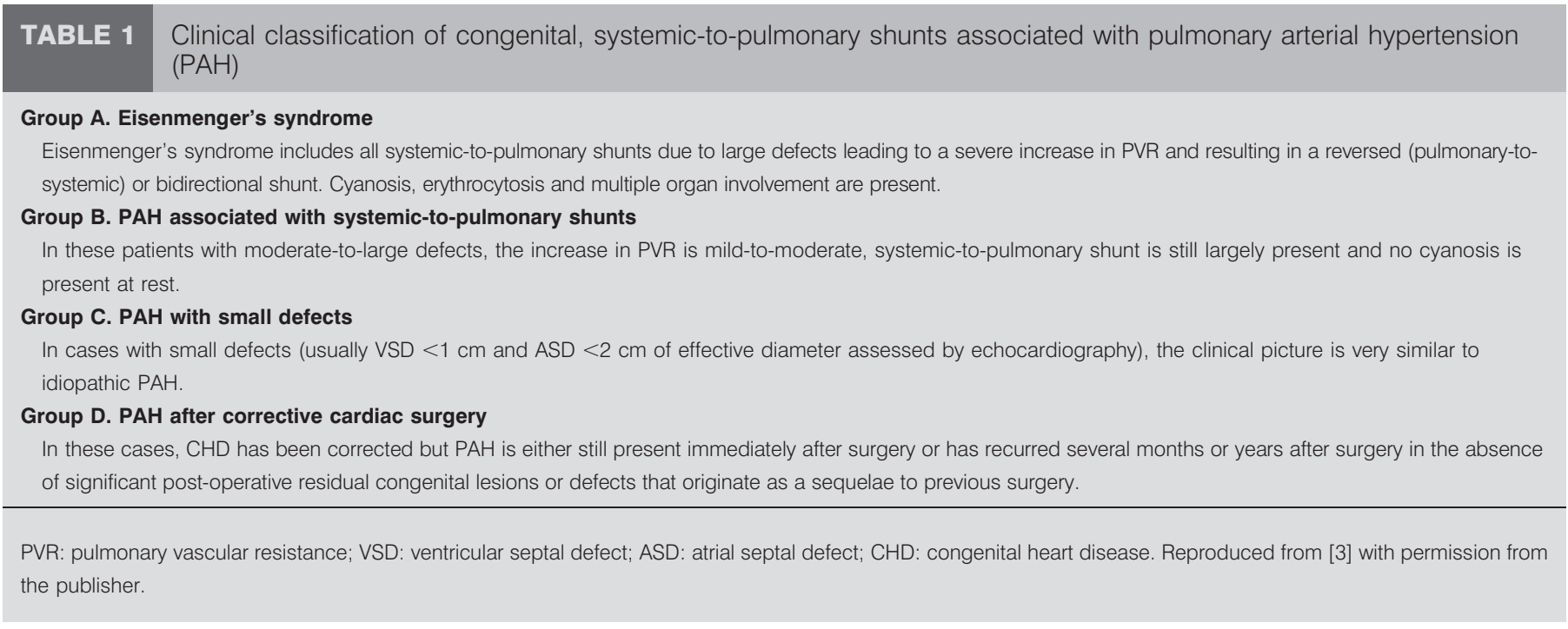

acts as a "relief valve" for the right heart, in effect acting as a "natural septostomy" [4]. Finally, there is a small group of patients (Group D) who have undergone corrective surgery, but who still have, or go on to develop, PAH despite the lack of any residual shunt. This is presumably because changes in their pulmonary vasculature had already progressed to a stage at which they had become irreversible or progressive despite correction. Group D patients have a poorer prognosis than patients with uncorrected PAH-CHD [21], which has led to concerns about repairing $\mathrm{CHD}$ in patients with overt $\mathrm{PAH}$. Although mortality rates in PAH-CHD are frequently reported as being more favourable than for other PAH aetiologies, it is important to remember that the patients who develop PAH are already survivors (i.e. a prevalent rather than incident population) and, therefore, there could be an unavoidable selection bias when comparing these patients with $\mathrm{PAH}-\mathrm{CHD}$ with patients with PAH associated with aetiologies. Despite some similarities, PAH-CHD does differ from other PAH aetiologies and, as discussed below, these differences may also contribute to the better prognosis associated with PAH-CHD compared with other PAH groups.

\section{PATHOPHYSIOLOGY OF PAH-CHD}

The timing of corrective surgery is critical to the avoidance of pulmonary vascular disease and PAH. The development of changes in the pulmonary arteries arising from persistently increased pulmonary pressure is a dynamic and multifactorial process, with progressive endothelial dysfunction leading to the characteristic vasoconstriction and remodelling of the pulmonary vascular bed [22]. Early changes to the pulmonary vasculature are likely to be reversible if the cardiac defect is repaired; patients with corrective surgery early in life (a few months of age) generally have normal PVR within 1 yr [23]. If surgery is delayed until later in childhood (after 2 yrs of age), PVR may fall post-surgery but normal levels may not be achieved [24]. Repair of an intracardiac lesion in the presence of established PAH may accelerate disease progression and the onset of right ventricular failure [23]. This suggests there may be a "point of no return" where changes in the pulmonary vasculature have progressed beyond a stage where full or partial reversibility is possible, despite correction of the associated defect. As will be discussed, this has implications for the decision to repair underlying defects in the presence of possible pulmonary vascular disease.

It is thought that PAH develops in CHD patients as a result of increased pulmonary blood flow due to the presence of a left-toright shunt. In particular, pre-tricuspid shunts lead to an overload volume in the pulmonary circulation, whereas posttricuspid shunts lead to a volume and pressure overload, resulting in early and more frequent development of $\mathrm{PAH}$. The shunt increases pressure in the pulmonary arteries leading to abnormal shear stress, circumferential wall stretch and endothelial dysfunction. Altered expression of vasoactive mediators, such as endothelin-1, prostacyclin and nitric oxide, results in vasoconstriction, while aberrant expression of vascular endothelial and fibroblast growth factors promotes vascular remodelling (including smooth muscle hypertrophy and proliferation) and increased intracellular matrix deposition [13]. Together these changes contribute to a progressive increase in PVR, with a concomitant increase in pressures in the right ventricle. If pressures in the right heart reach systolic vascular pressure, bidirectional shunting through the defect arises, with further increases in right atrial/ventricular pressure. The resulting reversal of the initial shunt to the right-to-left (pulmonary-tosystemic) shunt is characteristic of Eisenmenger's syndrome. However, compared with patients with other PAH aetiologies, the increases in pulmonary pressure seen in patients with $\mathrm{PAH}-$ CHD occur early (during infancy rather than during adulthood) and this seems to provide PAH-CHD patients with a prognostic advantage. It has been suggested that, during the early stages of life, the right ventricle can adapt in order to cope with the increased pressure; this early remodelling seems to allow the Eisenmenger's syndrome heart to accommodate a lifetime of increased pulmonary arterial pressure [25]. Interestingly, there are noticeable similarities between the Eisenmenger's syndrome heart and the fetal heart, which also has to function at much higher pulmonary artery pressures (PAPs) than the healthy adult heart [26].

The development of a right-to-left shunt in Eisenmenger's syndrome relieves the pressure in the right heart and preserves 
right ventricular function. However, this occurs with the consequence of cyanosis, the result of deoxygenated blood being returned to the systemic circulation, and an increased risk of a range of potentially life-threatening complications.

\section{COMORBIDITIES ASSOCIATED WITH PAH-CHD}

Patients with PAH-CHD suffer from a range of comorbidities associated with their underlying cardiac disorder, most commonly arrhythmias, heart failure, renal failure, hepatic dysfunction and diabetes mellitus [5, 27]. Arrhythmias, ranging from bradycardiac arrhythmias to ventricular tachycardia and atrial fibrillation, are common late complications of CHD. The development of atrial arrhythmia is associated with an increased risk of mortality due to heart failure-related death, sudden cardiac death and perioperative death in patients with $\mathrm{CHD}$, and $\mathrm{PAH}$ is an independent risk factor for mortality in these patients [28].

Eisenmenger's syndrome is a multisystem disorder and its development is associated with particularly high morbidity. Cyanosis leads to exercise intolerance and a range of potential problems including erythrocytosis, hyperviscosity, abnormalities of haemostasis, cerebral abscesses, stroke and endocarditis [29]. Cardiac complications include heart failure, syncope, cardiac arrhythmia and sudden death. Functional impairment is severe in adult CHD patients in general, with exercise capacity being depressed to levels seen in patients with chronic heart failure. The degree of this impairment is significantly greater in Eisenmenger's syndrome patients relative to other CHD patients [30]. Chronic hypoxaemia associated with Eisenmenger's syndrome also has numerous consequences. These include secondary polycythaemia, hyperviscosity, gout, joint and long bone pain as a result of hypertrophic osteoarthropathy, haemoptysis, and thrombosis [31].

The preservation of right ventricular function arising from the development of the right-to-left shunt means that patients with Eisenmenger's syndrome tend to live longer than those with idiopathic PAH, although the development of Eisenmenger's syndrome is still associated with reduced survival relative to CHD patients without PAH or those with PAH-CHD, but without Eisenmenger's syndrome [16]. A retrospective analysis of 188 patients over 31 yrs demonstrated that the most frequent causes of death in patients with Eisenmenger's syndrome were sudden unexpected death $(30 \%)$, heart failure $(23 \%)$ and massive haemoptysis (11\%), usually as a result of pulmonary artery rupture [32].

The multiple effects of their disease mean that Eisenmenger's syndrome patients have a particularly poor quality of life (QoL). As a result, management strategies for patients with Eisenmenger's syndrome should aim not only to improve outcome, but also QoL.

\section{TREATMENT OF PAH-CHD}

When considering therapy in patients with PAH-CHD, care needs to be taken to obtain a full and detailed history including chronological symptom presentation. This is particularly important in the assessment of functional class. Of all patients with CHD, those with Eisenmenger's syndrome have the most significant degree of functional impairment, with peak oxygen consumption close to that often used as the threshold for referral of other patients for transplantation. This can have a significant impact on the ability of Eisenmenger's syndrome patients to take part in day-to-day activities [30, 33]. However, because Eisenmenger's syndrome patients have often been living with their symptoms for many years, they tend to adapt their everyday activities to a lower intensity and often underestimate the degree to which their symptoms affect their activities [34]. This underestimation can affect their functional classification as classification relies on an assessment of how the patient's condition affects their "ordinary" activities. The perception of "ordinary" for a patient with long-standing Eisenmenger's syndrome may be very different from that of, for example, a patient with idiopathic $\mathrm{PAH}$ with a more recent onset of symptoms. It is, therefore, important when assessing CHD patients, and Eisenmenger's syndrome patients in particular, to ask a wide range of questions covering typical everyday activities to which the patient can easily relate in order to gain a true insight into their functional capabilities [35].

Current guidelines recommend that CHD patients requiring PAH-specific therapy are managed in specialised centres. Although some data on the use of PAH-specific therapies are available, particularly for Eisenmenger's syndrome patients, guidelines are generally based on the clinical experience of experts rather than formal evidence from clinical trials [3].

\section{General management of PAH-CHD}

Behavioural modification and awareness of potential risk factors are critical to the management of patients with $\mathrm{PAH}-$ CHD. General measures include avoidance of strenuous exercise, although mild activity is beneficial, and prevention of dehydration. Patients with Eisenmenger's syndrome are at particular risk during anaesthesia and surgery, and special care is required. Pregnancy is contraindicated in patients with Eisenmenger's syndrome as there is a high risk of maternal and fetal mortality; adequate contraception is, therefore, mandatory. Long-term supplemental oxygen therapy at home may improve symptoms. However, as this has not been shown to modify survival, at least when given only at night [36], the use of supplemental oxygen therapy is recommended only in cases in which it produces a consistent increase in arterial oxygen saturation and reduces symptoms [3].

Patients with Eisenmenger's syndrome are at an increased risk of coagulation disorders. The high incidence of pulmonary artery thrombosis (up to 20\%) is associated with increasing age, biventricular dysfunction and dilatation of the pulmonary arteries [37]. However, the use of anticoagulants in this population is controversial as there is also an increased risk of haemoptysis and haemorrhage [38]. Given the lack of data, guidelines do not recommend the use of anticoagulants in PAH-CHD, but suggest they may be considered in patients with pulmonary artery thrombosis, signs of heart failure and absent or mild haemoptysis [3]. Routine phlebotomy should not be performed as secondary erythrocytosis is beneficial for oxygen transport and delivery [3]. If moderate-to-severe symptoms of hyperviscosity are present, and iron deficiency and dehydration have been excluded, phlebotomy with isovolumic replacement should be performed carefully when the haematocrit is $>65 \%$ [38]. Iron deficiency has been shown to be associated with a higher risk of adverse outcomes (allcause mortality, transplantation and hospitalisation due to cardiopulmonary causes) in Eisenmenger's syndrome patients 
[39] and iron replacement therapy improves exercise tolerance and QoL [40]. However, care should be taken in patients with low oxygen saturations to avoid haemoglobin levels becoming too high [39]. There are no data to support the use of calcium channel blockers in patients with PAH-CHD and their use must be avoided [3]. In particular, their use is contraindicated in Eisenmenger's syndrome patients as this treatment class can result in an acute decrease in systemic arterial pressure and increase of the right-to-left shunt, which may lead to syncope and sudden death [20]. Patients who present with significant haemoptysis should be considered for embolisation of relevant collateral vessels if appropriate (fig. 2) [41].

\section{Management of PAH-CHD with PAH-specific therapies}

Reflecting available data, current European Society of Cardiology guidelines focus on patients with Eisenmenger's syndrome and recommend that treatment with the endothelin receptor antagonist bosentan is initiated in Eisenmenger's syndrome patients in functional class III (class I, level of evidence B), with consideration being given to the use of other endothelin receptor antagonists, phosphodiesterase type-5 inhibitors, prostanoids or combination therapy (class IIa, level of evidence C) [38]. This difference in recommendation level between bosentan and the other PAH-specific therapies occurs because only one randomised controlled trial of $\mathrm{PAH}$-specific therapies has been conducted and this involved bosentan. The BREATHE-5 (Bosentan Randomised Trial of Endothelin Antagonist-5) trial and its long-term open-label extension study demonstrated the benefit of bosentan in patients with Eisenmenger's syndrome in terms of significant improvements in exercise capacity, haemodynamics and functional class compared with placebo, independently of the location of the septal defect [42-44]. Data from a number of studies have since confirmed these benefits in the longer term in patients with PAH-CHD and specifically in patients with Eisenmenger's syndrome [45-50]. Importantly, treatment with bosentan has been shown not to reduce systemic arterial blood oxygen saturation over short- [42] and long-term treatment [47], demonstrating that it had no negative effect on the overall shunt. Treatment with bosentan has also been shown to have a positive long-term effect on QoL, a particularly important consideration for Eisenmenger's syndrome patients
[49]. Despite the lack of randomised controlled trials for other PAH-specific therapies, data are available from small, open-label studies; the earliest showing treatment benefit with epoprostenol [51]. Treatment with sildenafil has been shown to improve exercise capacity, Borg dyspnoea score, functional class, QoL and haemodynamics in patients with PAH-CHD/Eisenmenger's syndrome and appears to be well tolerated [52-57], while a single study of ambrisentan suggests that this endothelin receptor antagonist may also be beneficial in this patient population [58]. There is clearly an interest in using $\mathrm{PAH}-$ specific treatments in patients with $\mathrm{PAH}-\mathrm{CHD}$, but without further randomised controlled studies being initiated, evidence will continue to be based on single centre studies.

Although potentially attractive, results from studies investigating the benefits of combination therapy using different classes of PAH-specific therapy in PAH-CHD are mixed. While some studies suggest that the addition of sildenafil to bosentan therapy may improve haemodynamics and exercise capacity [27], others have shown no benefit [59]. However, overall, data suggest that treatment of PAH-CHD patients with PAH-specific therapy improves outcome. In a retrospective, single-centre analysis including 229 patients with Eisenmenger's syndrome, the use of PAH-specific therapies (advanced therapy: bosentan $73.5 \%$; sildenafil $25 \%$; epoprostenol $1.5 \%$ ) was associated with a significantly lower rate of cumulative mortality over 7 yrs versus no therapy [60]. To adjust for differences in clinical and demographic characteristics on advanced therapy and those not receiving this form of treatment, propensity scores were calculated with the use of logistic regression with advanced therapy as the dependent variable, and the baseline demographic and clinical variables as independent variable. Each patient on advanced therapy was matched with three patients not on advanced therapy and 10 matched populations were generated (fig. 3). Long-term PAH-specific therapy in patients with Eisenmenger's syndrome has been shown to improve both objective exercise capacity and subjective symptoms, although escalation of therapy over time may be required if symptoms deteriorate during treatment [61]. There are also data to show that PAH-specific therapies increase QoL scores in patients with PAH-CHD [49].
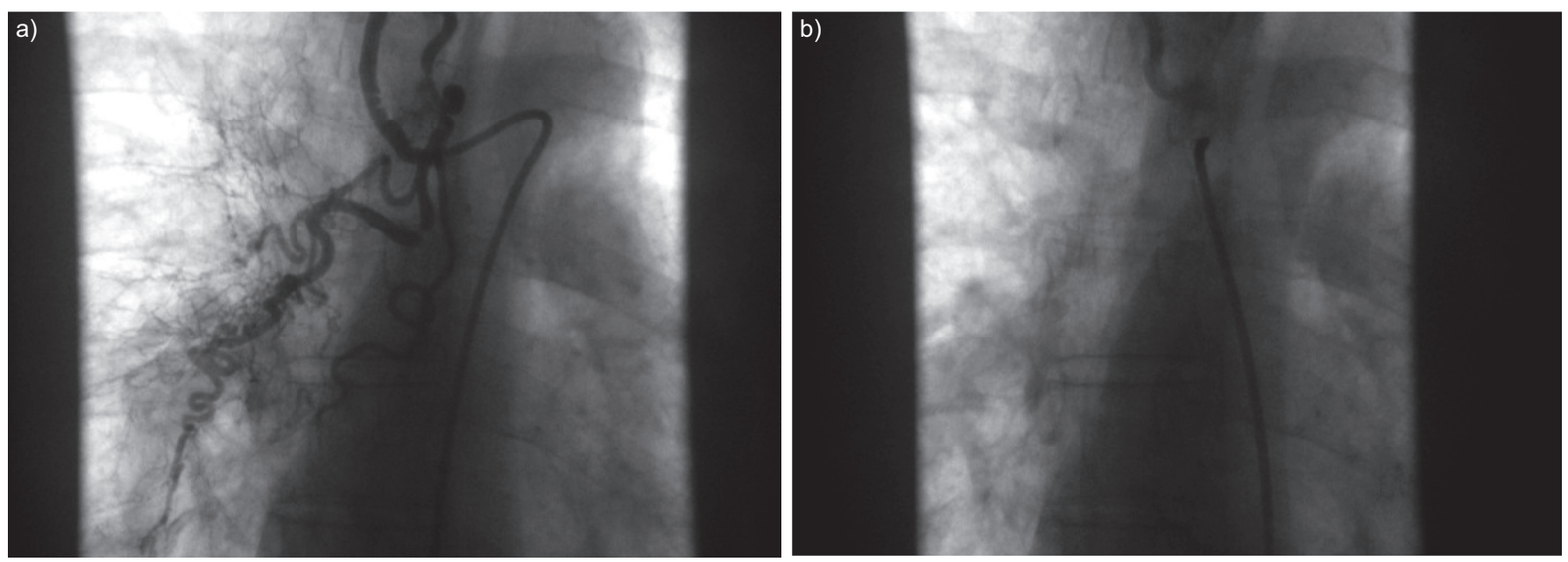

FIGURE 2. Angiogram of pulmonary collaterals in a patient with untreated aortopulmonary window during childhood. a) Before and b) after embolisation. 


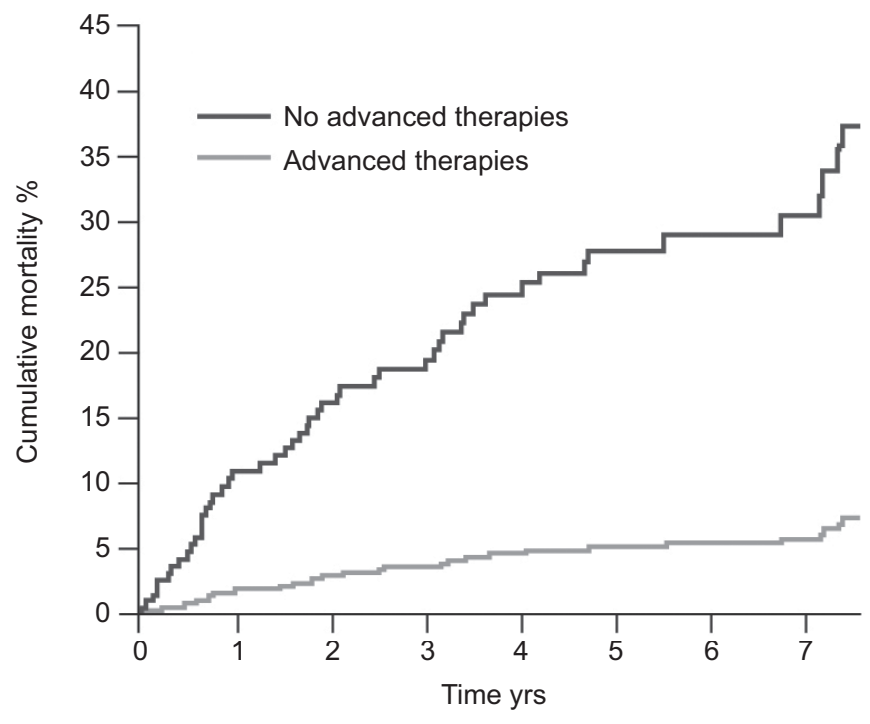

FIGURE 3. Adjusted survival rate curves, based on the propensity score model, of Eisenmenger's syndrome patients with and without advanced therapy (bosentan, sildenafil or epoprostenol). Quartiles of propensity score were based on the average propensity scores from the 10 matched populations. $p=0.015$ Reproduced from [60] with permission from the publisher.

Heart and lung transplantation is a potential treatment option for patients with PAH-CHD, but this is limited by the scarcity of donor organs in developed countries and the lack of organ specificity in developing countries. Although not studied extensively, as a result of the limitations mentioned above, it is possible that the comorbidities experienced by patients with PAH-CHD may affect the success of transplantation in this patient population.

\section{"TREAT-TO-CLOSE" CONSIDERATIONS}

Correction of an underlying congenital heart defect in infancy can prevent the development of PAH-CHD; however, a proportion of patients with left-to-right shunts are not recognised until later in their life, when they already have changes to the pulmonary vasculature and increased PVR. In those patients with increased PAP and Qp, but with a PVR within normal limits or only slightly raised, pulmonary vascular changes are likely to be minimal and the patient may benefit from surgery [62]. Conversely, those patients with high PAP and high PVR are likely to have extensive changes to the pulmonary vasculature and corrective surgery is contraindicated. There remains, therefore, a population of patients with medium-tolarge defects and moderate increases in PVR in whom the extent of pulmonary vascular changes and their potential to be reversed are unknown and so, in whom, the benefits or otherwise of corrective surgery are unclear. Currently there are no established markers of reversibility, although a number of candidates have been proposed. To date, the most promising marker is the number of circulating endothelial cells. This is a noninvasive marker of vascular damage and remodelling, which has been shown to be significantly raised in CHD patients with irreversible $\mathrm{PAH}$ post-surgery [63]. Further studies are required to confirm whether this is an appropriate marker. There are no evidence-based algorithms to guide assessment for operability in these patients, and decisions need to be made based on careful evaluation of the individual patient.

There has been interest in the potential for PAH-specific therapies to be used in a "treat-to-close" strategy, to reduce PVR and so improve the operability of patients with PAH-CHD. According to recently published Grown-Up Congenital Heart (GUCH) guidelines [38], patients with significant interatrial shunt $\left(Q_{p} / Q_{s}>1.5\right.$ or signs of right ventricular volume overload) and PVR $<5$ Wood units should undergo ASD closure (possibly percutaneously) regardless of symptoms (recommendation class I, level of evidence B). A weaker recommendation for closure (recommendation class $\mathrm{Ilb}$, level of evidence $\mathrm{C}$ ) is given for patients with $A S D, Q_{p} / Q_{s}>1.5$ and PVR $\geqslant 5$ Wood units, but less than two-thirds of systemic vascular resistance (SVR) or PAP less than two-thirds systemic pressure (baseline or when challenged with vasodilators, preferably nitric oxide, or after PAH-specific therapy). Similarly, patients with VSD are ideal candidates for closure if Qp/Qs is $>1.5$ and PVR is normal $(<5$ Wood units). They should be considered for closure (recommendation class Ilb, level of evidence C) when there is still net left-to-right shunt (Qp/Qs $>1.5)$ present and PAP or PVR are less than two-thirds of systemic values (baseline or when challenged with vasodilators, preferably nitric oxide, or after PAH-specific therapy).

Based on current knowledge, BEGHETTI et al. [62] suggest a PVR $<6$ Wood units together with a PVR/SVR ratio of 0.3 following 12 months of PAH-specific therapy as the haemodynamic upper limit for operability. However, data are lacking and clinical trials are needed to determine whether shunt closure, even where pre-operative PVR is reduced to within "safe" limits (yet to be defined), is ultimately the best option for these patients [17].

\section{PAH-CHD IN DOWN'S SYNDROME}

Down's syndrome (trisomy 21) is the most common chromosomal abnormality, with an estimated incidence of approximately 1.1 per 1,000 live births [64]. The incidence has been increasing in recent years [65]. Over the past few decades there has been a substantial increase in the life expectancy of children with Down's Syndrome, from around 12 yrs in the 1940s [66] to $35 \mathrm{yrs}$ in the 1980s [67] to $60 \mathrm{yrs}$ today [68, 69]. Down's syndrome is strongly associated with the development of pulmonary hypertension, predominantly associated with upper airway obstruction and CHD. The prevalence of CHD in neonates with Down's syndrome is around 42-58\%, with AVSD and VSD representing the most common defects $[9,69-$ 71]. A number of factors in individuals with Down's syndrome may contribute to the high rates of $\mathrm{PAH}$ reported in this population. Down's syndrome patients tend to have a high prevalence of the complex defects that are most commonly associated with PAH and, in particular, with Eisenmenger's syndrome. In addition, upper airway obstruction resulting from a range of pathologies, including tracheal stenosis and nasopharyngeal, oropharyngeal and subglottic compromise, is a common finding in patients with Down's syndrome, which may also contribute to the high prevalence of PAH in Down's syndrome patients [72]. There is also a high prevalence of pulmonary hypertension of the neonate among Down's syndrome patients, with an incidence of $1.2-5.2 \%$ in Down's 


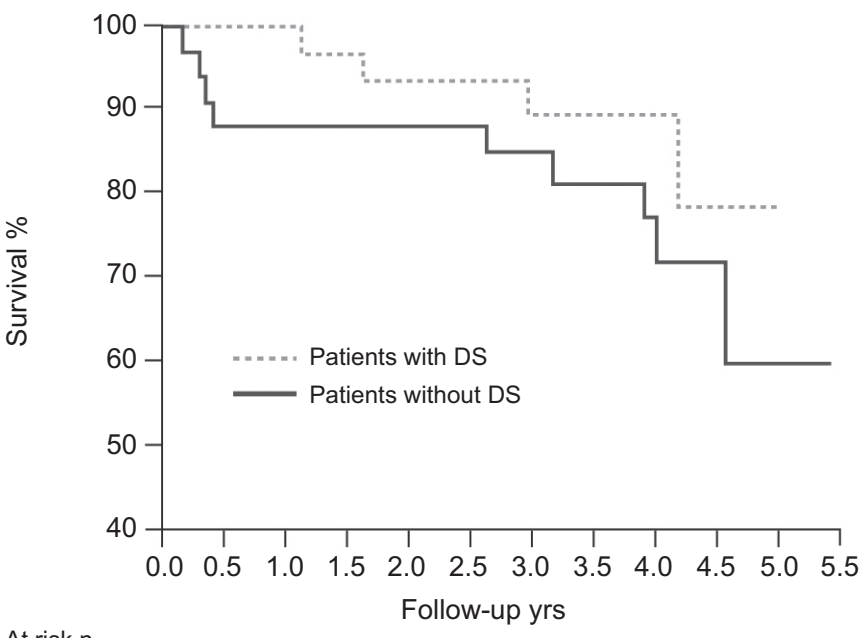

At risk $n$

$\begin{array}{llllll}\text { Without DS } & 34 & 29 & 27 & 21 & 9 \\ \text { With DS } & 34 & 30 & 27 & 20 & 8\end{array}$

FIGURE 4. 4-yr survival on bosentan in pulmonary arterial hypertensioncongenital heart disease patients with and without Down's syndrome (DS). $p=0.2$. Reproduced from [49] with permission from the publisher.

syndrome neonates compared with the reported general incidence of $0.1 \%[69,73]$. The reason for this is unclear.

As with Eisenmenger's syndrome patients without Down's syndrome, those with Down's syndrome have markedly reduced long-term survival, although there is no apparent difference in survival between Eisenmenger's syndrome patients with and without Down's syndrome [74]. However, when compared with other CHD patients, those with Down's syndrome are more prone to developing $\mathrm{PAH}$ earlier, probably due to a subtle endothelial dysfunction [75], and have worse functional capacity; both factors being associated with poorer long-term outcome $[9,60,76]$. Despite this, however, a recent registry study found that patients with Down's syndrome received significantly less PAH-specific treatment [9]. This may reflect the lack of clinical trials in Eisenmenger's syndrome in general, and in Eisenmenger's syndrome patients with Down's syndrome in particular, at the time the registry was initiated. Although there are no randomised controlled trials in Down's syndrome patients with PAH-CHD, data from open-label studies have emerged recently. Duffels et al. [77] reported stabilisation of the 6-min walk distance (6MWD) and QoL in Down's syndrome patients with PAH-CHD following a median of 22 months' bosentan therapy, although this was in contrast with a significant improvement in parameters in patients with PAH-CHD without Down's syndrome in the same study. VIs et al. [49] reported stabilisation of exercise capacity, stroke volume and QoL in Down's syndrome patients with PAH-CHD over 4 yrs of bosentan treatment, while significant improvements were seen in patients without Down's syndrome. Although mortality remained high, there was no significant difference in mortality between patients with and without Down's syndrome: $1-, 2-$ and 4-yr survival rates were $88 \%$, $88 \%$ and $72 \%$, respectively, in patients without Down's syndrome and $97 \%, 93 \%$ and $80 \%$, respectively, in patients with Down's syndrome (fig. 4) [49]. A further study found no

\begin{tabular}{|c|c|c|c|c|c|c|}
\hline & \multicolumn{3}{|c|}{ With Down's syndrome ${ }^{\#}$} & \multicolumn{3}{|c|}{ Without Down's syndrome ${ }^{\pi}$} \\
\hline & Basal & Follow-up & p-value & Basal & Follow-up & p-value \\
\hline \multicolumn{7}{|l|}{ Clinical status } \\
\hline WHO functional class & $2.9 \pm 0.6$ & $2.5 \pm 0.5$ & 0.005 & $2.9 \pm 0.5$ & $2.5 \pm 0.5$ & 0.000002 \\
\hline \multicolumn{7}{|l|}{ Exercise tolerance (6MWT) } \\
\hline Distance travelled $\mathrm{m}$ & $239 \pm 74$ & $288 \pm 71$ & 0.0007 & $343 \pm 86$ & $389 \pm 80$ & 0.00003 \\
\hline Heart rate at end of exercise beats. $\mathrm{min}^{-1}$ & $117 \pm 19$ & $109 \pm 9$ & 0.03 & $117 \pm 19$ & $111 \pm 20$ & 0.04 \\
\hline $\mathrm{Sp}, \mathrm{O}_{2}$ at end of exercise \% & $72 \pm 10$ & $74 \pm 10$ & 0.011 & $69 \pm 13$ & $73 \pm 12$ & 0.005 \\
\hline $\mathrm{mPpcw} \mathrm{mmHg}$ & $11 \pm 3$ & $12 \pm 3$ & 0.16 & $11 \pm 3$ & $12 \pm 3$ & 0.9 \\
\hline$Q p L \cdot m^{-1} \cdot m^{-2}$ & $3.5 \pm 1.4$ & $4.0 \pm 1.6$ & 0.006 & $2.8 \pm 1.0$ & $3.5 \pm 1.4$ & 0.0005 \\
\hline Qs $L \cdot m^{-1} \cdot m^{-2}$ & $3.6 \pm 1.3$ & $3.4 \pm 1.7$ & 0.37 & $3.3 \pm 1.3$ & $3.7 \pm 1.9$ & 0.064 \\
\hline$Q p / Q s$ & $1.0 \pm 0.4$ & $1.4 \pm 0.7$ & 0.003 & $0.9 \pm 0.3$ & $1.1 \pm 0.7$ & 0.012 \\
\hline PVRI Wood units & $20 \pm 13$ & $15 \pm 9$ & 0.007 & $26 \pm 15$ & $20 \pm 10$ & 0.002 \\
\hline
\end{tabular}

Data are presented as mean $\pm \mathrm{SD}$, unless otherwise stated. $\mathrm{Sp}, \mathrm{O}_{2}$ : arterial oxygen saturation measured by pulse oximetry; WHO: World Health Organization; $6 \mathrm{MWT}$ : 6-min walk test; Pra: right atrial pressure; mPAP: mean pulmonary artery pressure; mPpcw: mean pulmonary capillary wedge pressure; Qp: pulmonary flow; Qs: systemic flow; PVRI: pulmonary vascular resistance index. ${ }^{*}: n=18 ;{ }^{\bullet}: n=56$. Reproduced from [48] with permission from the publisher. 
differences in the efficacy of bosentan between PAH-CHD patients with and without Down's syndrome, with both groups showing significant improvements in 6MWD, World Health Organization functional class and haemodynamics at the end of 12 months of treatment (table 2) [48] without significant worsening of oxygen saturation [47]. In the latter study, the authors did not perform an analysis of change in 6MWD or QoL over time, reflecting the challenges associated with assessing treatment effect in patients with Down's syndrome. The validity of 6MWD in these patients has been disputed as walking distance has been shown to be inversely related to degree of intellectual disability in Down's syndrome patients [70]. The assessment of functional class may also pose problems, and it is important to get both the patient and carer's opinion of their functional limitations, while QoL questionnaires have not been properly validated in patients with Down's syndrome [9]. Assessment of patients with PAHCHD and Down's syndrome, particularly in the absence of haemodynamic data, is therefore challenging and requires special consideration. Although the lack of clinical trials in Down's syndrome patients with PAH-CHD contributes to the low rates of treatment initiation in this patient population, other factors will also play a part, not least the challenges of tailoring a management programme to meet the needs of each individual patient. As has been discussed previously, 6MWD is frequently not a valid indicator of cardiorespiratory fitness in adult patients with Down's syndrome and, therefore, in practice, conclusions about treatment efficacy may have to be based on variables other than those used as primary end-points in clinical studies. This makes it difficult to judge whether the change in parameter is of clinical relevance, particularly as the threshold of significance to the patient may vary considerably between individuals. It is clear that patients with Down's syndrome are entitled to treatment; however, we still seem to be a long way away from having all the information required in order to make appropriate management decisions for each individual patient.

\section{CONCLUSIONS}

Improvements in the diagnosis of $\mathrm{CHD}$ and its surgical and medical management have led to a significant increase in the number of patients surviving into adulthood. The best therapy for PAH-CHD remains prevention through a "timely" repair of the defect. The development of $\mathrm{PAH}$, and particularly Eisenmenger's syndrome, in these patients is associated with increased morbidity and mortality. There is increasing evidence of the benefits of PAH-specific therapy in PAH-CHD in terms of improving exercise capacity, symptoms and survival. Nevertheless, mortality remains relatively high, and there is a need for new therapeutic options and strategies in this complex patient population. Specific issues exist with regards to the management of certain patient subgroups. For example, the assessment of operability and timing of corrective surgery in patients with complex defects and increased pulmonary pressures warrants further investigation, particularly with regards to the use of $\mathrm{PAH}$-specific therapy as part of a treatto-close strategy. Patients with Down's syndrome make up a significant proportion of the PAH-CHD population, but historically have not been managed optimally. There is a lack of clinical studies in these patients and, although new and encouraging data are emerging, there remains a need for further investigations. Given the difficulties associated with standard measures of prognosis such as functional class and
$6 \mathrm{MWD}$, there is a need for the establishment of new prognostic indicators and tailored QoL questionnaires to better evaluate treatment effect in these patients.

Overall, we are beginning to see progress in the management and treatment of patients with PAH-CHD; however, much still needs to be done to address the many issues involved in the management of this growing and complex patient group.

\section{STATEMENT OF INTEREST}

V.S. Mahadevan has received a research grant from Actelion Pharmaceuticals.

\section{ACKNOWLEDGEMENTS}

We received editorial assistance from L. Quine (Elements Communications Ltd, Westerham, UK) supported by Actelion Pharmaceuticals Ltd (Allschwil, Switzerland).

\section{REFERENCES}

1 Eisenmenger V. Die angeborenen Defecte der Kammerscheidewand des Herzens. Z Klin Med 1897; 32: 1-28.

2 Wood P. The Eisenmenger syndrome or pulmonary hypertension with reversed central shunt. I. Br Med J 1958; 2: 701-709.

3 Galiè N, Hoeper MM, Humbert M, et al. Guidelines for the diagnosis and treatment of pulmonary hypertension: the Task Force for the Diagnosis and Treatment of Pulmonary Hypertension of the European Society of Cardiology (ESC) and the European Respiratory Society (ERS), endorsed by the International Society of Heart and Lung Transplantation (ISHLT). Eur Heart J 2009; 30: 2493-2537.

4 Galiè N, Manes A, Palazzini M, et al. Management of pulmonary arterial hypertension associated with congenital systemic-topulmonary shunts and Eisenmenger's syndrome. Drugs 2008; 68: 1049-1066.

5 Rodriguez FH 3rd, Moodie DS, Parekh DR, et al. Outcomes of hospitalization in adults in the United States with atrial septal defect, ventricular septal defect, and atrioventricular septal defect. Am J Cardiol 2011; 108: 290-293.

6 Lowe BS, Therrien J, Ionescu-Ittu R, et al. Diagnosis of pulmonary hypertension in the congenital heart disease adult population impact on outcomes. J Am Coll Cardiol 2011; 58: 538-546.

7 Report of the British Cardiac Society Working Party. Grown-up congenital heart $(\mathrm{GUCH})$ disease: current needs and provision of service for adolescents and adults with congenital heart disease in the UK. Heart 2002; 88: Suppl. 1, i1-i14.

8 Diller GP, Gatzoulis MA. Pulmonary vascular disease in adults with congenital heart disease. Circulation 2007; 115: 1039-1050.

9 Van de Bruaene A, Delcroix M, Pasquet A, et al. The Belgian Eisenmenger syndrome registry: implications for treatment strategies? Acta Cardiol 2009; 64: 447-453.

10 Brickner ME, Hillis LD, Lange RA. Congenital heart disease in adults: first of two parts. N Engl J Med 2000; 342: 256-263.

11 Kaemmerer H, Bauer U, de Haan F, et al. Recommendations for improving the quality of the interdisciplinary medical care of grown-ups with congenital heart disease (GUCH). Int J Cardiol 2011; 150: 59-64.

12 Marelli AJ, Mackie AS, Ionescu-Ittu R, et al. Congenital heart disease in the general population: changing prevalence and age distribution. Circulation 2007; 115: 163-172.

13 Adatia I, Kothari SS, Feinstein JA. Pulmonary hypertension associated with congenital heart disease: pulmonary vascular disease: the global perspective. Chest 2010; 137: Suppl. 6, 52S-61S.

14 Duffels MG, Engelfriet PM, Berger RM, et al. Pulmonary arterial hypertension in congenital heart disease: an epidemiologic perspective from a Dutch registry. Int J Cardiol 2007; 120: 198-204. 
15 Humbert M, Sitbon O, Chaouat A, et al. Pulmonary arterial hypertension in France: results from a national registry. Am J Respir Crit Care Med 2006; 173: 1023-1030.

16 Engelfriet PM, Duffels MG, Möller T, et al. Pulmonary arterial hypertension in adults born with a heart septal defect: the Euro Heart Survey on adult congenital heart disease. Heart 2007; 93: 682-687.

17 Beghetti M, Galiè N. Eisenmenger syndrome a clinical perspective in a new therapeutic era of pulmonary arterial hypertension. J Am Coll Cardiol 2009; 53: 733-740.

18 Collins-Nakai RL, Rabinovitch M. Pulmonary vascular obstructive disease. Cardiol Clin 1993; 11: 675-687.

19 Vongpatanasin W, Brickner ME, Hillis LD, et al. The Eisenmenger syndrome in adults. Ann Intern Med 1998; 128: 745-755.

20 Diller GP, Dimopoulos K, Broberg CS, et al. Presentation, survival prospects and predictors of death in Eisenmenger's syndrome: a combined retrospective and case-control study. Eur Heart J 2006; 27: $1737-1742$.

21 van Loon RL, Roofthooft MT, Hillege HL, et al. Pediatric pulmonary hypertension in the Netherlands: epidemiology and characterization during the period 1991 to 2005. Circulation 2011; 124: $1755-1764$.

22 Humbert M, Sitbon O, Simonneau G. Treatment of pulmonary arterial hypertension. N Engl J Med 2004; 351: 1425-1436.

23 Haworth SG. Pulmonary hypertension in the young. Heart 2002; 88: 658-664.

24 Balint OH, Samman A, Haberer K, et al. Outcomes in patients with pulmonary hypertension undergoing percutaneous atrial septal defect closure. Heart 2008; 94: 1189-1193.

25 Hopkins WE. The remarkable right ventricle of patients with Eisenmenger syndrome. Coron Artery Dis 2005; 16: 19-25.

26 Hopkins WE, Waggoner AD. Severe pulmonary hypertension without right ventricular failure: the unique hearts of patients with Eisenmenger syndrome. Am J Cardiol 2002; 89: 34-38.

27 D'Alto M, Romeo E, Argiento P, et al. Bosentan-sildenafil association in patients with congenital heart disease-related pulmonary arterial hypertension and Eisenmenger physiology. Int J Cardiol 2012; 155: 378-382.

28 Yap SC, Harris L, Chauhan VS, et al. Identifying high risk in adults with congenital heart disease and atrial arrhythmias. Am J Cardiol 2011; 108: 723-728.

29 DeFilippis AP, Law K, Curtin S, et al. Blood is thicker than water: the management of hyperviscosity in adults with cyanotic heart disease. Cardiol Rev 2007; 15: 31-34.

30 Diller GP, Dimopoulos K, Okonko D, et al. Exercise intolerance in adult congenital heart disease: comparative severity, correlates, and prognostic implication. Circulation 2005; 112: 828-835.

31 Somerville J. How to manage the Eisenmenger syndrome. Int $J$ Cardiol 1998; 63: 1-8.

32 Daliento L, Somerville J, Presbitero P, et al. Eisenmenger syndrome. Factors relating to deterioration and death. Eur Heart J 1998; 19: 1845-1855.

33 Kempny A, Dimopoulos K, Uebing A, et al. Reference values for exercise limitations among adults with congenital heart disease. Relation to activities of daily life-single centre experience and review of published data. Eur Heart J 2012; 33: 1386-1396.

34 Dimopoulos K, Giannakoulas G, Wort SJ, et al. Pulmonary arterial hypertension in adults with congenital heart disease: distinct differences from other causes of pulmonary arterial hypertension and management implications. Curr Opin Cardiol 2008; 23: 545-554.

35 Raphael C, Briscoe C, Davies J, et al. Limitations of the New York Heart Association functional classification system and selfreported walking distances in chronic heart failure. Heart 2007; 93: 476-482.

36 Sandoval J, Aguirre JS, Pulido T, et al. Nocturnal oxygen therapy in patients with the Eisenmenger syndrome. Am J Respir Crit Care Med 2001; 164: 1682-1687.
37 Broberg CS, Ujita M, Prasad S, et al. Pulmonary arterial thrombosis in Eisenmenger syndrome is associated with biventricular dysfunction and decreased pulmonary flow velocity. J Am Coll Cardiol 2007; 50: 634-642.

38 Baumgartner $\mathrm{H}$, Bonhoeffer $\mathrm{P}$, De Groot $\mathrm{N}$, et al. ESC guidelines for the management of grown-up congenital heart disease (new version 2010). Eur Heart J 2010; 31: 2915-2957.

39 Van De Bruaene A, Delcroix M, Pasquet A, et al. Iron deficiency is associated with adverse outcome in Eisenmenger patients. Eur Heart J 2011; 32: 2790-2799.

40 Tay EL, Peset A, Papaphylactou M, et al. Replacement therapy for iron deficiency improves exercise capacity and quality of life in patients with cyanotic congenital heart disease and/or the Eisenmenger syndrome. Int J Cardiol 2011; 151: 307-312.

41 Mamas MA, Clarke B, Mahadevan VS. Embolisation of systemicto-pulmonary collaterals in patients with the Eisenmenger reaction presenting with haemoptysis. Cardiol Young 2008; 18: 528-531.

42 Galiè N, Beghetti M, Gatzoulis MA, et al. Bosentan therapy in patients with Eisenmenger syndrome: a multicenter, double-blind, randomized, placebo-controlled study. Circulation 2006; 114: 48-54.

43 Gatzoulis MA, Beghetti M, Galiè N, et al. Longer-term bosentan therapy improves functional capacity in Eisenmenger syndrome: results of the BREATHE- 5 open-label extension study. Int J Cardiol 2008; 127: 27-32.

44 Berger RM, Beghetti M, Galiè N, et al. Atrial septal defects versus ventricular septal defects in BREATHE-5, a placebo-controlled study of pulmonary arterial hypertension related to Eisenmenger's syndrome: a subgroup analysis. Int J Cardiol 2010; 144: 373-378.

45 Diller GP, Dimopoulos K, Kaya MG, et al. Long-term safety, tolerability and efficacy of bosentan in adults with pulmonary arterial hypertension associated with congenital heart disease. Heart 2007; 93: 974-976.

46 Díaz-Caraballo E, González-García AE, Reñones M, et al. Longterm bosentan treatment of complex congenital heart disease and Eisenmenger's syndrome. Rev Esp Cardiol 2009; 62: 1046-1049.

47 Monfredi O, Griffiths L, Clarke B, et al. Efficacy and safety of bosentan for pulmonary arterial hypertension in adults with congenital heart disease. Am J Cardiol 2011; 108: 1483-1488.

48 D'Alto M, Romeo E, Argiento P, et al. Therapy for pulmonary arterial hypertension due to congenital heart disease and Down's syndrome. Int J Cardiol 2011; [Epub ahead of print DOI: 10.1016/ j.ijcard.2011.07.009].

49 Vis JC, Duffels MG, Mulder P, et al. Prolonged beneficial effect of bosentan treatment and 4-year survival rates in adult patients with pulmonary arterial hypertension associated with congenital heart disease. Int J Cardiol 2011; [Epub ahead of print DOI: 10.1016/ j.ijcard.2011.06.064].

50 Kaya MG, Lam YY, Erer B, et al. Long-term effect of bosentan therapy on cardiac function and symptomatic benefits in adult patients with Eisenmenger syndrome. J Card Fail 2012; 18: 379-384.

51 Rosenzweig EB, Kerstein D, Barst RJ. Long-term prostacyclin for pulmonary hypertension with associated congenital heart defects. Circulation 1999; 99: 1858-1865.

52 Lu XL, Xiong CM, Shan GL, et al. Impact of sildenafil therapy on pulmonary arterial hypertension in adults with congenital heart disease. Cardiovasc Ther 2010; 28: 350-355.

53 Tay EL, Papaphylactou M, Diller GP, et al. Quality of life and functional capacity can be improved in patients with Eisenmenger syndrome with oral sildenafil therapy. Int J Cardiol 2011; 149: 372-376.

54 Zhang ZN, Jiang $X$, Zhang R, et al. Oral sildenafil treatment for Eisenmenger syndrome: a prospective, open-label, multicentre study. Heart 2011; 97: 1876-1881.

55 Chau EM, Fan KY, Chow WH. Effects of chronic sildenafil in patients with Eisenmenger syndrome versus idiopathic pulmonary arterial hypertension. Int J Cardiol 2007; 120: 301-305. 
56 Zeng WJ, Lu XL, Xiong CM, et al. The efficacy and safety of sildenafil in patients with pulmonary arterial hypertension associated with the different types of congenital heart disease. Clin Cardiol 2011; 34: 513-518.

57 Mukhopadhyay S, Sharma M, Ramakrishnan S, et al. Phosphodiesterase-5 inhibitor in Eisenmenger syndrome: a preliminary observational study. Circulation 2006; 114: 1807-1810.

58 Zuckerman WA, Leaderer D, Rowan CA, et al. Ambrisentan for pulmonary arterial hypertension due to congenital heart disease. Am J Cardiol 2011; 107: 1381-1385.

59 Iversen $\mathrm{K}$, Jensen AS, Jensen TV, et al. Combination therapy with bosentan and sildenafil in Eisenmenger syndrome: a randomized, placebo-controlled, double-blinded trial. Eur Heart J 2010; 31: 1124-1131.

60 Dimopoulos K, Inuzuka R, Goletto S, et al. Improved survival among patients with Eisenmenger syndrome receiving advanced therapy for pulmonary arterial hypertension. Circulation 2010; 121: 20-25.

61 Diller GP, Alonso-Gonzalez R, Dimopoulos K, et al. Disease targeting therapies in patients with Eisenmenger syndrome: response to treatment and long-term efficiency. Int J Cardiol 2012; [Epub ahead of print DOI: 10.1016/j.jicard.2012.02.007].

62 Beghetti M, Galiè N, Bonnet D. Can "inoperable" congenital heart defects become operable in patients with pulmonary arterial hypertension? Dream or reality? Congenit Heart Dis 2012; 7: 3-11.

63 Smadja DM, Gaussem P, Mauge L, et al. Circulating endothelial cells: a new candidate biomarker of irreversible pulmonary hypertension secondary to congenital heart disease. Circulation 2009; 119: 374-381.

64 Morris JK, Alberman E. Trends in Down's syndrome live births and antenatal diagnoses in England and Wales from 1989 to 2008: analysis of data from the National Down Syndrome Cytogenetic Register. BMJ 2009; 339: b3794.

65 Weijerman ME, de Winter JP. Clinical practice. The care of children with Down syndrome. Eur J Pediatr 2010; 169: 1445-1452.

66 Penrose LS. The incidence of Mongolism in the general population. J Ment Sci 1949; 95: 685-688.
67 Thase ME. Longevity and mortality in Down's syndrome. J Ment Defic Res 1982; 26: 177-192.

68 Glasson EJ, Sullivan SG, Hussain R, et al. The changing survival profile of people with Down's syndrome: implications for genetic counselling. Clin Genet 2002; 62: 390-393.

69 Weijerman ME, van Furth AM, van der Mooren MD, et al. Prevalence of congenital heart defects and persistent pulmonary hypertension of the neonate with Down syndrome. Eur J Pediatr 2010; 169: 1195-1199.

70 Vis JC, Thoonsen H, Duffels MG, et al. Six-minute walk test in patients with Down syndrome: validity and reproducibility. Arch Phys Med Rehabil 2009; 90: 1423-1427.

71 Irving CA, Chaudhari MP. Cardiovascular abnormalities in Down's syndrome: spectrum, management and survival over 22 years. Arch Dis Child 2012; 97: 326-330.

72 Shapiro NL, Huang RY, Sangwan S, et al. Tracheal stenosis and congenital heart disease in patients with Down syndrome: diagnostic approach and surgical options. Int $J$ Pediatr Otorhinolaryngol 2000; 54: 137-142.

73 Cua CL, Blankenship A, North AL, et al. Increased incidence of idiopathic persistent pulmonary hypertension in Down syndrome neonates. Pediatr Cardiol 2007; 28: 250-254.

74 Troost E, Van De Bruaene A, Lampropoulos K, et al. The outcome of Eisenmenger patients with trisomy 21 does not differ from patients without trisomy 21. Acta Cardiol 2011; 66: 293-301.

75 Cappelli-Bigazzi M, Santoro G, Battaglia C, et al. Endothelial cell function in patients with Down's syndrome. Am J Cardiol 2004; 94: 392-395.

76 Suzuki K, Yamaki S, Mimori S, et al. Pulmonary vascular disease in Down's syndrome with complete atrioventricular septal defect. Am J Cardiol 2000; 86: 434-437.

77 Duffels MG, Vis JC, Rosa LE, et al. Effect of bosentan on exercise capacity and quality of life of adults with pulmonary arterial hypertension associated with congenital heart disease with or without Downs syndrome. Am J Cardiol 2009; 103: 1309-1315. 